http://jmscr.igmpublication.org/home/

ISSN (e)-2347-176x ISSN (p) 2455-0450

crossref DOI: https://dx.doi.org/10.18535/jmscr/v9i4.13

Journal Of Medical Science And Clinical Research

\title{
Mean platelet volume: Significance in patients with IBD: Ulcerative Colitis
}

Authors

\author{
Anuvind T, I Shubha, Caroline Selvi K, K Premkumar, R Murali, A Chezhian
}

Institute of Medical Gastroenterology, Madras Medical College, The Tamil Nadu Dr. M.G.R Medical

University, Chennai, Tamil Nadu, India

\begin{abstract}
Background: Mean platelet volume (MPV) and inflammatory markers are affected in chronic inflammatory diseases. In this study we aimed to investigate the correlation of MPV with disease activity in patients with IBD: Ulcerative Colitis based on the UCEIS score. The UCEIS consists of the following three descriptors and was calculated as a simple sum: vascular pattern (scored 0-2); bleeding (scored 0-3); and erosions and ulcers (scored 0-3). Therefore, the range of UCEIS scores is 0-8.

Materials and Methods: A total of 120 subjects were enrolled. The study group consisted of 40 patients with Ulcerative Colitis and the control group included 80 healthy subjects. Groups were compared regarding MPV and disease activity score - UCEIS.

Results: Groups were similar in terms of age, sex ( $p=0.23,0.43$, respectively). MPV levels were higher in patients with UC patients $9.71 \pm 1.08$ compared to the controls $11.27 \pm 0.83(p=0.02)$. A significant correlation was found between MPV and UCEIS Score in patients.

Conclusion: $M P V$ was found to be decreased in patients with IBD compared to the controls and there is a negative correlation with disease activity indices.
\end{abstract}

\section{Introduction}

Ulcerative colitis is an immune mediated chronic inflammatory disease of the bowel which pursue a protracted relapsing and remitting course, usually extending over years. UC usually involves the rectum and extends proximally to involve all or part of the colon. Some patients present with mild disease activity and do well with generally safe and mild medications, but many others exhibit more severe disease. There are numerous instruments to measure disease activity, some of which are purely clinical, endoscopic, or histologic, and others that combine clinical and endoscopic assessments.

Platelets are the smallest and yet extremely reactive blood components. They are involved first of all in the processes of fibrosis and maintenance of haemostasis. Recent studies highlight on their multifunctional nature. ${ }^{1}$ Platelets are the first to accumulate at the site of damage, where they change in shape and show formation of pseudopodia, local release of cytoplasmic granular content, and aggregation, when activated by classical agonists, such as ADP, TXA2, PAF, and inflammatory cytokines, e.g., IL-1, IL-6, and TNF alpha. ${ }^{2}$ Platelets promotes inflammatory processes by secretion of biologically active substances like platelet activation factor, IL-1, platelets factor 4 . Platelet function correlates with their size MPV. ${ }^{3}$ The MPV has been investigated as a potential biomarker in IBD. ${ }^{4,5,6}$. The results of the research remain contradictory. The aim of this study is to investigate the significance of MPV in patients 
with Ulcerative colitis and correlation with disease activity.

\section{Objective}

To determine the relation between the disease activity of ulcerative colitis and mean platelet volume (MPV) and to evaluate the clinical usage of a more simple and easier determinant.

\section{Materials \& Methods}

A total of 120 subjects were enrolled during the study period from July 2019 to June 2020. The study group consisted of 40 patients of both sex, age ranging from 18-70 years with Ulcerative Colitis, and the control group included 80 healthy subjects. The informed consent was obtained from all participants in the study.

Patient data was collected prospectively using standardized data collection forms created specifically for this study. All patients had a definitive diagnosis of UC confirmed by clinical, radiological, endoscopic, and histological studies. None of the patients were under medication (such as oral anticoagulants, aspirin, nonsteroidal antiinflammatory drugs, and contraceptives)that might have caused platelet or coagulation abnormalities during the last 8 weeks before blood sampling. Patients with abnormal liver or renal function tests, myeloproliferative disorders, or malignancies were not included in the study.

\section{Laboratory Parameters}

Full blood count (including MPV and white blood cells (WBC)), CRP and ESR were performed on admission (before prescribing any medications). For platelet number and MPV measurement: platelet number (per mm3) and MPV (fentoliter (fL), blood samples were taken from forehand into EDTA tubes by applying a minimal stasis from antecubital vein.

For other parameters, the measurement and process were carried out according to standard laboratory practice. To calculate the body mass index, height and weight were recorded on admission for each individual. For patients, the disease activity was defined according to the Ulcerative colitis endoscopic index of severity.

All variables were stated as mean \pm standard deviation. For statistical analysis, the relation between MPV and variables was examined through Pearson and Spearman correlation. $\mathrm{P}<0.05$ value was accepted as statistically significant

\section{Results}

9 of 40 ulcerative colitis patients were having ulcerative proctitis (E1) (22.5\%), 18(45\%) patients were having Left sided colitis (E2), and 13 pancolitis (32.5\%) 9 were in remission (22.5 $\%)$ and 31 of them had active disease $(77.5 \%)$

Details of the clinical characteristics of the study patients are shown in Table 1. Disease activity in patients with UC was assessed by the Ulcerative Colitis Endoscopic Index of Severity (UCEIS), which incorporates three scores (Vascular, Haemorrhage, Erosions and Ulcers). Demographic data including age, sex, year of diagnosis, age at diagnosis, smoking status, and a family history of inflammatory bowel disease (IBD) as well as annual measurements of disease severity and course of disease were recorded.Groups were compared regarding MPV, platelet count, CRP, ESR.

The demographic features of UC patients and healthy controls are shown in Table 1. The distributions of age, gender, smoking habit and body mass index were not statistically significant between groups

Table 2 demonstrates comparisons of all inflammatory markers, including MPV, CRP and ESR. A significant reduction in MPV was noted in patients with ulcerative colitis compared with healthy controls $(9.71 \pm 1.08 \mathrm{fl}$ vs. $11.27 \pm 0.83 \mathrm{fl}$, $\mathrm{P}=0.02)$. Meanwhile, CRP $(15.6 \pm 2.8 \mathrm{mg} / \mathrm{dl}$ vs. $1.9 \pm 0.96 \mathrm{mg} / \mathrm{dl}, \mathrm{P}<0.003)$ and $\operatorname{ESR}(25.67 \pm$ $1.87 \mathrm{~mm} /$ hour vs. $8.83 \pm 1.72 \mathrm{~mm} /$ hour, $\mathrm{P}<0.009$ ) were statistically higher in the ulcerative colitis group than those in the control group. 
Table 1 Demographics of ulcerative colitis patients and control group

\begin{tabular}{|l|c|c|c|}
\hline Characteristics & Ulcerative Colitis $(\mathrm{n}=40)$ & Control $(\mathrm{n}=80)$ & $\mathrm{P}$ value \\
\hline Age & $46.6 \pm 1.36$ & $49.5 \pm 1.84$ & 0.23 \\
\hline Sex: Male & $22(55 \%)$ & $41(51.25 \%)$ & 0.43 \\
\hline Smoking & $11(27.5 \%)$ & $23(28.75 \%)$ & 0.73 \\
\hline BMI & $20.3 \pm 0.982$ & $21.32 \pm 0.527$ & 0.13 \\
\hline Socioeconomic status & $8.93 \pm 1.06$ & $9.8 \pm 1.63$ & 0.39 \\
\hline Hypertension & $5(12.5 \%)$ & $9(11.25 \%)$ & 0.10 \\
\hline Diabetes Mellitus & $4(10 \%)$ & $9(11.25 \%)$ & 0.49 \\
\hline
\end{tabular}

Table 2 Comparison of MPV and other inflammatory markers between Ulcerativecolitis and control group

\begin{tabular}{|l|c|c|c|}
\hline & Cases & Control & P- value \\
\hline MPV & $9.71 \pm 1.08$ & $11.27 \pm 0.83$ & 0.02 \\
\hline CRP & $15.6 \pm 2.8$ & $1.9 \pm 0.96$ & 0.003 \\
\hline ESR & $25.67 \pm 1.87$ & $8.83 \pm 1.72$ & 0.009 \\
\hline
\end{tabular}

We further investigated the ability of MPV and other markers in differentiating UC patients from healthy controls.A significant correlation was found between MPV and IBD disease activity, which was measured with UCEIS score in UC. ${ }^{7}$

Using the Spearman coefficient we analysed the co-relation between Mean Platelet Volume and disease severity using the Ulcerative Colitis Endoscopic Index of Severity (UCEIS)and found that there is a significant negative correlation between MPV and disease severity $(\mathrm{r}=-0.7 \mathrm{P}=$ 0.004)

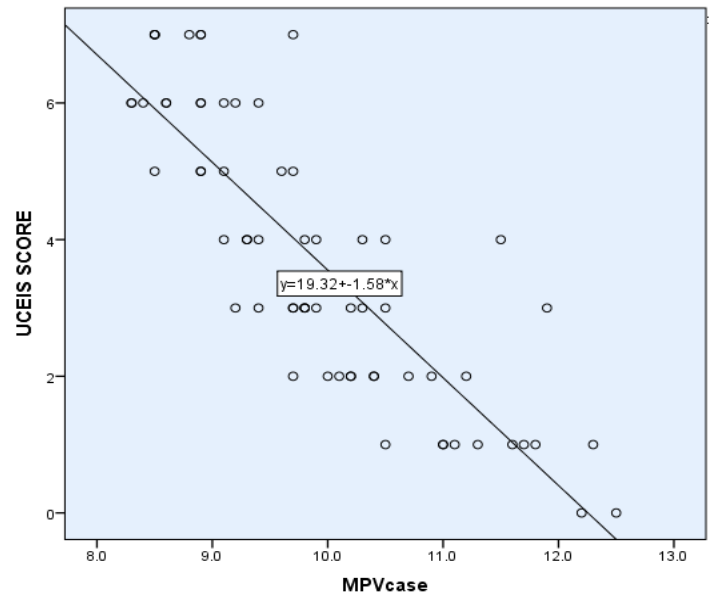

\section{Discussion}

Previous studies have demonstrated that appropriate and effective therapy can control symptoms, maintain remission, prevent relapse, improve quality of life and reduce mortality in patients with Ulcerative Colitis. Therefore, early diagnosis and assessment of disease activity is essential for tailoring therapy. ${ }^{8}$ As invasive techniques, including endoscopic, radiological and histopathologic methods, are routinely used for diagnostic decision and disease activity monitoring, an ideal non-invasive test is increasingly expected for initial diagnosis and identification of disease activity. ${ }^{9}$

The mean platelet volume (MPV) reflects the size of platelets. It can be measured during a routine automatic whole blood count. The MPV has been shown to correlate with the function and activation of platelets. ${ }^{3}$ The importance of MPV has been emphasized as an inflammation marker in some chronic inflammatory disorders, such as inflammatory intestinal diseases, rheumatoid arthritis, and ankylosing spondylitis. ${ }^{10}$ An inverse correlation between disease activity and MPV has been demonstrated. Several stool tests, such as faecal lactoferrin, calprotectin and elastase, were investigated as novel inflammatory markers for IBD. ${ }^{11}$ Even though they are superior to CRP or ESR with higher sensitivity and specificity in detecting gastrointestinal inflammation, they are not specific markers for IBD. Small platelets have lower functional capabilities. ${ }^{12}$ This may in part explain the bleeding frequently seen in IBD patients with active disease. According to one of the hypotheses, the decrease of platelet volume observed in CD and UC could occur due to the presence of a disorder in the regulation of thrombopoiesis often associated with systemic inflammation. $^{13}$ 
The correlation between MPV and inflammation has been well investigated in the literature. Previous series of case-control and cohort studies have documented several essential confounders of MPV. Smoking and exposure to nicotine were key factors that would significantly influence platelet morphology and size, and could be confounded by sex, age and duration of smoking. In our study, the age, sex and smoking histories were statistically similar between healthy controls and patients with UC. ${ }^{14}$

Mustafa Güçlü et al showed that there is negative significant co-relations between MPV and clinical, pathological and colonoscopic activity indices. ${ }^{15}$ However, there was no relation between the extent of the disease involvement and MPV. Kapsoritakis et al collected data for 66 patients with Crohns Disease and 38 healthy volunteers, and found a negative correlation of MPV with CRP, ESR and WBC. Ya Levin et al study demonstrates that there is increase in platelet and erythrocyte aggregation, accompanied by the decrease in their volume and increase in erythrocyte anisocytosis. ${ }^{17}$ The results of our study are not in concordance with the case control study done by Tayyibe Saler et al who recruited 200 subjects (100 patients with IBD (UC: 50 and CD:50) and the control group which included 100 healthy Subjects) could not find any significant association between MPV and inflammatory bowel disease. ${ }^{18}$ Song Liu et al demonstrated that MPV was low in Crohns disease patients compared with healthy subjects, but MPV did not show a discriminative value in disease activity. ${ }^{19}$ Platelets play a crucial role in the pathogenesis of IBD. The statistically significant reduction in MPV in patients with Ulcerative Colitis observed in our study is in accordance with findings of most of the prior studies, suggesting that MPV may be helpful in clinical practice, especially to the primary care physicians in differentiating inflammatory diarrhoea from functional aetiologies and also predicting the severity of the disease. According to one of the hypotheses, the reduction of platelet volume seen in patients with
UC could be due to the presence of a disorder in the regulation of thrombopoiesis often associated with systemic inflammation. Another hypothesis is that itcould occur due to quicker consumption of the large activated platelets. Another factor that can explain the decrease in MPV could be enhanced blood cells microvesiculation. ${ }^{20}$ According to literature, a substantial amount of Microvesicles (MV) in blood is derived from the platelet. The release of micro vesicles results in decrease of the MPV. MVs are known to play a part in pathogenesis of multiple conditions, especially associated with the risk of thrombosis ${ }^{21}$

\section{Conclusion}

MPV was found to be decreased in patients with IBD compared to the controls and there is a negative correlation with disease activity indices.Our study uncovered a preliminary but inspiring finding that Mean Platelet Volume could be used as a good biomarker in distinguishing Ulcerative colitis from healthy controls. Nevertheless, we are also aware that our finding is not sufficient for employing MPV solely in this differential diagnosis but our finding could provide a suggestion for physicians to seek attention to the value of MPV and take it into account while making decisions of differential diagnosis. Our study is limited by small study sample so further studies with large sample size are needed to validate the above findings

\section{References}

1. Öztürk ZA, Dag MS, Kuyumcu ME, Cam H, Yesil Y, Yilmaz N, et al. Could platelet indices be new biomarkers for inflammatory bowel diseases? Eur Rev Med Pharmacol Sci. 2013;17(3):334-41.

2. Talstad I, Rootwelt K, Gjone E. Thrombocytosis in ulcerative colitis and Crohn's disease. Scand J Gastroenterol 1973;8: 135-8

3. Bath PM, Butterworth RJ. Platelet size: measurement, physiology and vascular 
disease. Blood Coagul Fibrinolysis. 1996; 7(2):157-61.

4. Öztürk ZA, Dag MS, Kuyumcu ME, Cam H, Yesil Y, Yilmaz N, et al. Could platelet indices be new biomarkers for inflammatory bowel diseases? Eur Rev Med Pharmacol Sci. 2013;17(3):334-41.

5. Clarke K, Sagunarthy R, Kansal S. RDW as an Additional Marker in Inflammatory Bowel Disease/Undifferentiated Colitis. Dig Dis Sci. 2008;53(9):2521-3.

6. Danese S, Motte Cde L, Fiocchi C. Platelets in inflammatory bowel disease: clinical, pathogenic, and therapeutic implications. Am J Gastroenterol. 2004;99(5):938-45.

7. Travis SP, Schnell D, Krzeski P, et al. Developing an instrument to assess the endoscopic severity of ulcerative colitis: the Ulcerative Colitis Endoscopic Index of Severity (UCEIS). Gut 2012;61:535-542.

8. Peyrin-Biroulet L, Pane's J, Sandborn WJ, et al. Defining Disease Severity in InflammatoryBowel Diseases: Current and Future Directions. Clin GastroenterolHepatol 2016;14(3):34854.e17.

9. Lewis JD. The utility of biomarkers in the diagnosis and therapy of inflammatory bowel disease. Gastroenterology 2011;140(6):1817-26.e2.

10. Semple JW, Italiano JE, Freedman J. Platelets and the immune continuum. Nat Rev Immunol 2011;11(4):264-74. doi: 10.1038/nri2956.

11. Schoepfer AM, Beglinger C, Straumann A, et al. Fecal calprotectin more accurately reflects endoscopic activity of ulcerative colitis than the lichtiger index, C-reactive protein, platelets, hemoglobin, and blood leukocytes. Inflamm Bowel Dis 2013;19(2):332-41.

12. Camitta BM, Slye RJ. Optimizing Use of the Complete Blood Count PediatriaPolska 2012;87(1):72-7.
13. Kamath S, Blann AD, Lip GY. Platelet activation: assessment and quantification. Eur Heart J. 2001;22(17):1561-71.

14. Gasparyan AY, Ayvazyan L, Mikhailidis DP, Kitas GD: Mean platelet volume: a link between thrombosis and inflammation? Curr Pharm Des 2011, 17:47-58.Danese S, Motte Cde L, Fiocchi C. Platelets in inflammatory bowel disease: clinical, pathogenic, and therapeutic implications. Am J Gastroenterol. 2004;99(5):938-45.

15. Mustafa Guclu, HakanSakallı, TolgaYakar. Mean Platelet Volume may be Reflects the Disease Activity of Ulcerative Colitis. Eur J Gen Med 2010; 7: 259-263

16. Kapsoritakis AN, Koukourakis MI, Sfiridaki A, PotamianosSP, Kosmadaki MG, Koutroubakis IE, Kouroumalis EA.Mean platelet volume: a useful marker of inflammatory bowel disease activity. Am J Gastroenterol 2001; 96: 776-781

17. Levin, G., Popovicheva, A.N., Sosnina, L., Fedulova, E.N., \&Sheremet'ev, Y.A. (2018). Mean Red Cell and Platelet Volume and Blood Cells Aggregation in Children with Inflammatory Bowel Diseases.

18. Saler, T., ÖzgürKeşkek, Ş., Ergin, C., Ahbab, S. and Sağlam, Z., 2016. Mean platelet volume in patients with inflammatory bowel disease. General Internal Medicine and Clinical Innovations, 1(3), pp.40-43.

19. Liu S, Ren J, Han G, Wang G, Gu G, Xia Q, Li J. Mean platelet volume: a controversial marker of disease activity in Crohn's disease. Eur J Med Res 2012; 17: 27

20. Deutschmann A, Schlagenhauf A, Leschnik B, Hoffmann KM, Hauer A, Muntean W. Increased procoagulant function of microparticles in pediatric inflammatory bowel disease: role in 
increased thrombin generation. J Pediatr Gastroenterol Nutr. 2013;56(4):401-7.

21. Preston RA, Jy W, Jimenez JJ, Mauro LM, Horstman LL, Valle M, et al. Effects of severe hypertension on endothelial and platelet microparticles. Hypertension. 2003;41(2):211-7. 